\title{
Forward Roll Coating of a Williamson's Material onto a Moving Web: A Theoretical Study
}

\author{
M. Zahid, ${ }^{1}$ I. Siddique $\mathbb{D}^{2},{ }^{2}$ S. Saleem $\mathbb{D}^{3},{ }^{3}$ A. Al-Zubaidi, ${ }^{3}$ M. A. Rana, ${ }^{4}$ and M. Zafar ${ }^{4}$ \\ ${ }^{1}$ Department of Mathematics, COMSATS University Islamabad, Abbottabad Campus, Abbottabad 22060, Pakistan \\ ${ }^{2}$ Department of Mathematics, University of Management and Technology, Lahore 54770, Pakistan \\ ${ }^{3}$ Department of Mathematics, College of Science, King Khalid University, Abha 61413, Saudi Arabia \\ ${ }^{4}$ Department of Mathematics and Statistics, Riphah International University, Islamabad, Pakistan \\ Correspondence should be addressed to I. Siddique; imransmsrazi@gmail.com
}

Received 13 May 2021; Revised 14 July 2021; Accepted 14 August 2021; Published 23 August 2021

Academic Editor: Jianguo Wang

Copyright $\odot 2021 \mathrm{M}$. Zahid et al. This is an open access article distributed under the Creative Commons Attribution License, which permits unrestricted use, distribution, and reproduction in any medium, provided the original work is properly cited.

\begin{abstract}
This paper presents a mathematical model for the thin film roll coating process of an incompressible Williamson material, passing through a closed passage between a rotating roll and a web. In light of lubrication approximation theory, the flow equations are nondimensionalized. The regular perturbation approach is used to provide solutions for the velocity profile, pressure gradient, flow rate per unit width, and shear stress at the roll surface. Important engineering quantities such as coating thickness, maximum pressure, separation point, roll/sheet separating force, and roll-transmitted power to the fluid are also obtained. The effects of several factors are graphically projected. The study shows that the material factors that are involved determine the operating variables. Coating thickness and separation point are controlled by Weissenberg's number, therefore acting as a controlling parameter for the rate of flow, thickness in coating, power contribution, pressure, roll separating force, and separation point. In comparison to the existing results in the literature, the current results are broader and zero-order results are more accurate.
\end{abstract}

\section{Introduction}

Deposition of fluid to the substrate under consideration is performed through roll coating in an industrial process. The phenomenon had gained a healthy reputation during the past few decades. As an example, the paper coating, decoration, or protection of fabrics or metal with such materials, films, coated products and magnetic recording, thin uniform liquid coating are the examples of coating in industry. To carry out these operations, a variety of apparatuses are used, in which the most common are roll coaters. The flow of nonNewtonian materials has several industrial and physiological applications in many coatings processes. Moreover, such flows have caused various challenges for mathematical scientists and model developers in the development of procedures suitable for the calculation of flows. This is due to the complexity and higher-order nature of leading flow expressions of non-Newtonian fluids than the Navier-Stokes equations.
Due to the complex nature of flows, numerous leading expressions for non-Newtonian flows are suggested in the literature. Abegunrin et al. [1] analyzed the boundary layer flow of two non-Newtonian fluids over an upper horizontal surface of a paraboloid of revolution. Abegunrin and Animasaun [2] studied the motion of non-Newtonian Williamson fluid over an upper horizontal surface of a paraboloid of revolution due to partial slip and buoyancy. Shah et al. [3] signified the water base nanoparticle's radius, heat flux due to concentration gradient, and mass flux due to the temperature gradient. Animasaun and Omowaye [4] studied the upper-convected Maxwell fluid flow with variable thermophysical properties over a melting surface situated in a hot environment subject to thermal stratification. Stability analysis of convection non-Newtonian vertical fluid layer in the presence of gold nanoparticles is performed by Mekheimer et al. [5]. Zaher et al. [6] studied the residual time of sinusoidal metachronal ciliary flow of non-Newtonian fluid through ciliated walls. Koumy et al. [7] 
investigated the hall and porous boundaries effects on peristaltic transport through a porous medium of a Maxwell model. Abdelsalam et al. [8] theoretically investigated the physical traits of electromagnetohydrodynamics (EMHD) of the bloodstream in the presence of electroosmotic forces via arteries having both aneurysm and stenosis for a hybrid fractional second-grade nanofluid. Raza et al. [9] investigated the thermal transport of radiative Williamson fluid over the stretchable curved surface. Pseudoplastic fluids are considered the most common in non-Newtonian fluids. Studies of pseudoplastic fluids have gained excessive attention for their utmost utility in industrial processes like extruding of polymer films, melts, and solutions with high molecular weight polymers and films coated with emulsion such as photographic films. Due to the pseudoplastic nature of Williamson fluid [10-12], its model is being focused on. However, less consideration has been paid to the Williamson fluid model, which describes the properties of pseudoplastic fluids. Nadeem and Akbar [13] debated the peristaltic flow of a Williamson fluid. Cramer and Marchello [14] demonstrated with experimental evidence that this model is better suited to polymer solutions and particle suspensions than other models.

For better understanding the procedure and to foresee the complications faced during the development, many hypothetical surveys have been undertaken on the flows under discussion. Model for flow conduct inside toner of the printing press by considering the concept of lubrication was developed by Taylor and Zeltlemoyer [15]. The values for force and pressure distribution were calculated by them. Hintermaier and White [16] developed the model for the water flow between two rolls by using lubrication theory and observed the results compatible with their conclusions carried out experimentally. Moreover, the flow theory of roll coating of Newtonian Liquid, assuming the traditional lubrication concepts and some general physical concepts was developed by Greener and Middleman [17]. Their model was modified by Benkreira et al. [18] to a general situation of two rollers of equal or unequalized rotating at the same speed or uneven one, consequently establishing a model related to the general situation. Their conclusions were found close to experimental results. Numerical outcomes on the mentioned problem over a moving flat subs rate were provided by Sofou [19] undertaking the lubrication approximation theory. They manipulated the Herschel-Bulkley model of viscoplasticity, thereby reducing it to the Newtonian models under suitable modifications, Bingham, and power-law. Recently, Zahid et al. [20, 21] undertook the same situation with the viscoelastic fluid being incompressible in which the roll, as well as web, was assumed to move with the same rates. To simplify expressions for motion, they performed the above-mentioned theory. Using the regular perturbation method, solutions for main flow, pressure gradient, flow rate per unit width, and shear stress at the roll surface are computed.

To the best of our knowledge, no study has been highlighted on the said fluid under roll coating in the mentioned works. The focus of the present study is to establish the flow technique for the model under consideration and to examine the influence of fluid physical properties on the coating process. Among physical properties, the Weissenberg number (We) is used in the study of viscoelastic flows and it usually gives the relation of stress relaxation time of the fluid and a specific process time. Some interesting studies regarding Weissenberg numbers are given in $[22,23]$. The paper has been ordered as below. In the upcoming section, the flow equations and the construction of the problem are defined. The succeeding part discusses the exact solution of the flow parameters. Lastly, outcomes, discussion, and deductions are offered.

\section{Mathematical Formulation}

Consider a steady, laminar flow of an incompressible Williamson fluid between a flat plate and cylindrical roll of radius $R$ as represented in Figure 1. The plate moves with a constant velocity $U$ in a positive $x$-direction, as the roll rotates counterclockwise with angular velocity $\omega$. The plate and cylinder have a gap at the nip, $H_{0}$. The polymer first bites the plane at the location $x=-x_{b}$, as shown in Figure 2. The expressions leading the flow of a said fluid are as follows:

$$
\begin{aligned}
& \nabla \cdot \mathbf{V}=0, \\
& \rho \frac{\mathrm{d} \mathbf{V}}{\mathrm{d} t}=-\nabla p+\nabla \cdot \tau,
\end{aligned}
$$

where $\mathbf{V}$ is the velocity, $\rho$ is the density, $p$ is the pressure, $\mathrm{d} / \mathrm{d} t(\cdot)=\partial / \partial t(\cdot)+\mathbf{V} \cdot \nabla(\cdot)$ is the material derivative, and $\tau$ is the extra stress tensor, for a Williamson fluid [24] defined as

$$
\tau=\left[\eta_{\infty}+\left(\eta_{0}-\eta_{\infty}\right)(1-\Gamma \widetilde{\gamma})^{-1}\right] \mathbf{A}_{1},
$$

where $\eta_{\infty}$ and $\eta_{0}$ are correspondingly the infinite and zero shear rate viscosities, respectively, $\mathbf{A}_{1}=\nabla \mathbf{V}+(\nabla \mathbf{V})^{t}$ is the first Rivlin-Ericksen tensor, $\Gamma$ is the time constant, and the shear rate $\widetilde{\gamma}[5]$ is defined as

$$
\tilde{\gamma}=\sqrt{\frac{1}{2} \sum_{i} \tilde{\gamma}_{i j} \sum_{j} \tilde{\gamma}_{j i}}=\sqrt{\frac{1}{2} \pi},
$$

where $\pi=\operatorname{trace}\left(\mathbf{A}_{1}^{2}\right)$ is the second invariant stress tensor. Constitutive equation (3) takes the following form, when we consider the case for which $\eta_{\infty}=0$ and $\Gamma \tilde{\gamma}<1$ :

$$
\tau=\eta_{0}(1+\Gamma \widetilde{\gamma}) \mathbf{A}_{1} .
$$

The above model reduces to Newtonian when $\Gamma=0$. The curved channel length molded by the plane and the roll is much larger than the parting at the nip, that is, $H_{0} \ll R$, thereby causing the flow to become two-dimensional. The velocity profile consequently takes the following form:

$$
\mathbf{V}=\left[u_{11}(x, y), v_{11}(x, y)\right] .
$$

In view of equation (6), the flow equations (1) and (2) in components form can be written as 


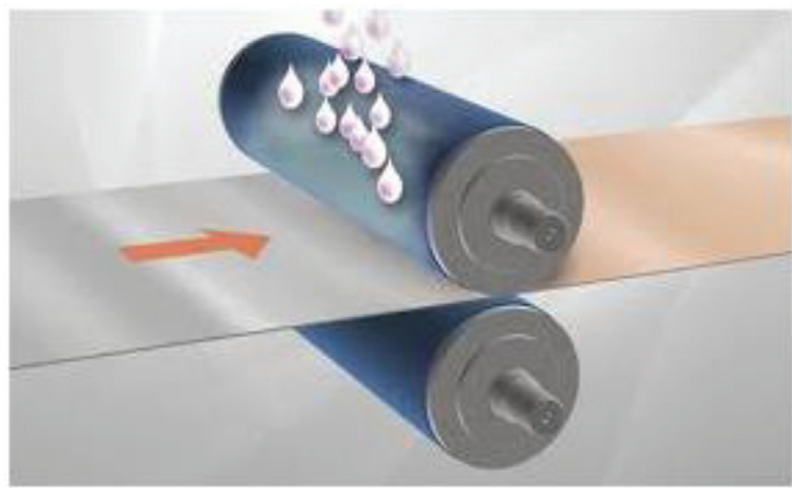

Figure 1: Physical representation of forward roll coating.

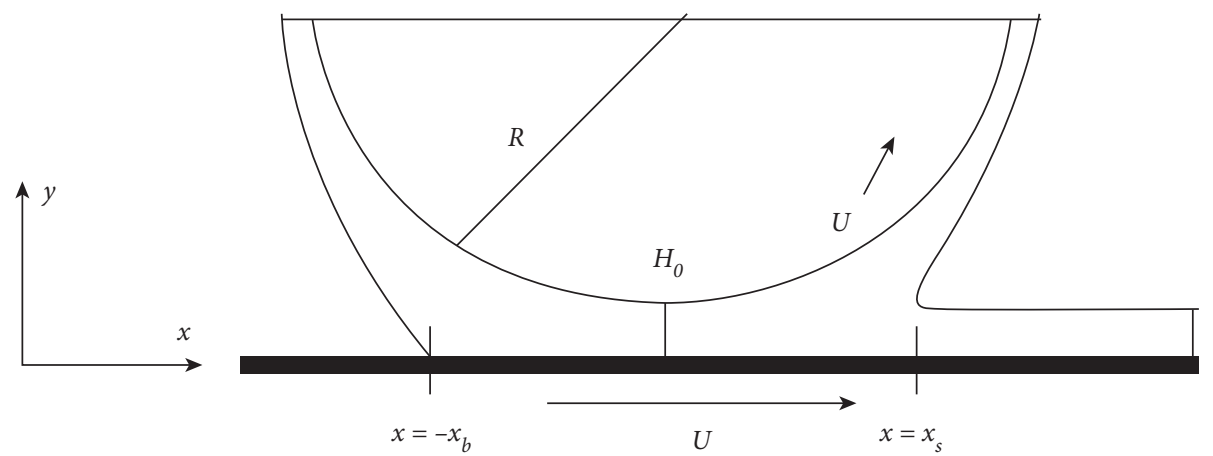

Figure 2: Geometry of the studied physical model.

$$
\begin{gathered}
\frac{\partial u_{11}}{\partial x}+\frac{\partial v_{11}}{\partial y}=0 \\
\rho\left(u_{11} \frac{\partial u_{11}}{\partial x}+u_{11} \frac{\partial v_{11}}{\partial y}\right)=-\frac{\partial p_{11}}{\partial y}+\frac{\partial \sigma_{x x}}{\partial x}+\frac{\partial \tau_{y x}}{\partial y} \\
\rho\left(u_{11} \frac{\partial v_{11}}{\partial x}+v_{11} \frac{\partial v_{11}}{\partial y}\right)=-\frac{\partial p_{11}}{\partial y}+\frac{\partial \tau_{x y}}{\partial x}+\frac{\partial \sigma_{y y}}{\partial y}
\end{gathered}
$$

where $\sigma_{x x}, \tau_{y x}, \tau_{x y}$, and $\sigma_{y y}$ are the stress components which can be written from equation (5) as

$$
\begin{aligned}
& \sigma_{x x}=2 \eta_{0}(1+\Gamma \tilde{\gamma}) \frac{\partial u_{11}}{\partial x} \\
& \tau_{x y}=\tau_{y x}=2 \eta_{0}(1+\Gamma \widetilde{\gamma})\left(\frac{\partial u_{11}}{\partial x}+\frac{\partial v_{11}}{\partial y}\right), \\
& \sigma_{y y}=2 \eta_{0}(1+\Gamma \tilde{\gamma}) \frac{\partial u_{11}}{\partial y} .
\end{aligned}
$$

We start with the LAT assumptions that the most significant dynamic phenomena happen in the region where the minimum gap exists, that is, the nip region. In that region, and extending to either side (i.e., the $\pm x$ direction) by a distance of the order of $x_{0}$, the roll surfaces are nearly parallel. Then, it is reasonable to assume that $v_{11} \ll u_{11}$ and $(\partial / \partial x) \ll(\partial / \partial y)$. In order to obtain the characteristic scale for the velocity and pressure, we conduct in brevity an order of magnitude analysis, and we can identify the following scales for $x, y$, and $u_{11}, x \sim L_{c}, y \sim H_{0}, u_{11} \sim U, u_{11} \sim U$. Then, from equation (1), we obtain $\left(v_{c} / U\right) \sim\left(H_{0} / L_{c}\right) \ll 1$, which shows that the order of magnitude of a transversal velocity $v_{c}$ is smaller than the longitudinal velocity, where the longitudinal characteristic length is given by $L_{c}=\sqrt{2 R H_{0}}$. Based on the above discussion, we introduce the following dimensionless quantities:

$$
\begin{aligned}
& \bar{x}=\frac{x}{\sqrt{R H_{0}}}, \\
& \bar{y}=\frac{y}{H_{0}}, \\
& \bar{u}=\frac{u_{11}}{U}, \\
& \bar{v}=\frac{v_{11}}{\vartheta_{1} U}, \\
& \bar{p}=\frac{p_{11} H_{0}}{\eta_{0} U} \sqrt{\frac{H_{0}}{R}}, \\
& \overline{\tilde{\gamma}}=\frac{\tilde{\gamma} H_{0}}{U}, \\
& \vartheta_{1}=\sqrt{\frac{H_{0}}{R}},
\end{aligned}
$$


into equations (7)-(10), and we get the following nondimensional equations (after dropping (-) for simplicity):

$$
\begin{gathered}
\frac{\partial u}{\partial x}+\frac{\partial v}{\partial y}=0 \\
\operatorname{Re}_{1}\left(u \frac{\partial u}{\partial x}+v \frac{\partial u}{\partial y}\right)=-\frac{\partial p}{\partial x}+\vartheta_{1} \frac{\partial \sigma_{x x}}{\partial x}+\frac{\partial \tau_{x y}}{\partial y} \\
\operatorname{Re}_{1}^{3}\left(u \frac{\partial v}{\partial x}+v \frac{\partial v}{\partial y}\right)=-\frac{\partial p}{\partial y}+\vartheta_{1} \frac{\partial \sigma_{y y}}{\partial y}+\vartheta_{1}^{2} \frac{\partial \tau_{x y}}{\partial y} \\
\sigma_{x x}=2 \vartheta_{1}[1+\mathrm{We} \tilde{\gamma}] \frac{\partial u}{\partial x} \\
\tau_{x y}=\tau_{y x}=[1+\mathrm{We} \tilde{\gamma}]\left(\frac{\partial u}{\partial y}+\vartheta_{1}^{2} \frac{\partial v}{\partial x}\right) \\
\sigma_{y y}=2 \vartheta_{1}[1+\mathrm{We} \tilde{\gamma}] \frac{\partial v}{\partial x}
\end{gathered}
$$

where $\operatorname{Re}=\rho U H_{0} / \eta_{0}, \mathrm{We}=\Gamma U / H_{0}$, and $\widetilde{\gamma}=\left[2 \vartheta_{1}^{2}(\partial u / \partial x)^{2}+\right.$ $\left.\left((\partial u / \partial y)+\vartheta_{1}^{2}(\partial v / \partial x)\right)^{2}+2 \vartheta_{1}^{2}(\partial v / \partial y)^{2}\right]^{(1 / 2)}$.

Neglecting the terms having $\vartheta_{1}$ being small quantity as $\vartheta_{1}$ is a square root of the ratio of $H_{0}$ to $R$, we get

$$
\begin{aligned}
\frac{\partial}{\partial y}\left\{\frac{\partial u}{\partial y}\left[1+\mathrm{We} \frac{\partial u}{\partial y}\right]\right\} & =\frac{\partial p}{\partial x} \\
\frac{\partial p}{\partial y} & =0 .
\end{aligned}
$$

It is obvious from equation (17) that $p$ is not a function of $y$, so it is the function of $x$ only; that is, $p=p(x)$. Thus, equation (16) becomes

$$
\frac{\mathrm{d} p}{\mathrm{~d} x}=\frac{\partial^{2} u}{\partial y^{2}}+\mathrm{We} \frac{\partial}{\partial y}\left[\left(\frac{\partial u}{\partial y}\right)^{2}\right] .
$$

The corresponding nondimensional boundary conditions are

$$
\left.\begin{array}{ll}
u=1, & \text { at } y=0, \\
u=1, & \text { at } y=h(x),
\end{array}\right\},
$$

where $h(x)=1+\left(x^{2} / 2\right)$ represents the dimensionless gap between roll and the web. Along with equation (18), the following nondimensional balanced mass equation is needed:

$$
\lambda=\int_{0}^{h(x)} u(y) \mathrm{d} y
$$

where $\lambda=\left(Q / U W H_{0}\right), Q$ is the dimensionless flow rate per unit width.

\section{Solution of the Problem}

Most of the mathematical problems do not possess explicit solutions, so it is very important to know methods for approximating the solution of such type of problems. Asymptotic analysis like the perturbation technique provides a powerful procedure for obtaining approximate solutions to complex problems. Such problems contain a parameter that is very large or very small, and one wants to take advantage of this structure to get the best approximation. These techniques are very useful in converging/diverging geometries like coating flow problems. The closed-form solution of equation (18) is difficult being a nonlinear differential equation. Therefore, regular perturbation is used to find its solution. We take We $\ll 1$ (as a perturbation parameter) and expand velocity $u$, pressure $p$, and dimensionless film thickness in power series of We:

$$
\begin{gathered}
u=u_{0}+\mathrm{We} u_{1}+\mathrm{We}^{2} u_{2}+\mathrm{O}\left(\mathrm{We}^{3}\right), \\
\frac{\mathrm{d} p}{\mathrm{~d} x}=\frac{\mathrm{d} p_{0}}{\mathrm{~d} x}+\mathrm{We} \frac{\mathrm{d} p_{1}}{\mathrm{~d} x}+\mathrm{We} \mathrm{e}^{2} \frac{\mathrm{d} p_{2}}{\mathrm{~d} x}+\mathrm{O}\left(\mathrm{We}^{3}\right), \\
\lambda=\lambda_{0}+\mathrm{We} \lambda_{1}+\mathrm{We}^{2} \lambda_{2}+\mathrm{O}\left(\mathrm{We}^{3}\right) .
\end{gathered}
$$

Substituting expressions (21)-(23) into equations (18)-(20) and equating like powers of We, we obtain the following problems.

3.1. Zeroth-Order Problem and Its Solution. Comparing terms free from We in equation (18), we have the following zero-order problem:

$$
\begin{aligned}
\frac{\mathrm{d} p_{0}}{\mathrm{~d} x} & =\frac{\mathrm{d}^{2} u}{\mathrm{~d} y^{2}}, \\
\lambda_{0} & =\int_{0}^{h(x)} u_{0} \mathrm{~d} y,
\end{aligned}
$$

with the agreeing zeroth-order boundary conditions:

$$
\begin{array}{ll}
u_{0}=1, & \text { at } y=0, \\
u_{0}=1, & \text { at } y=h(x) .
\end{array}
$$

The solution for equation (24) with conditions (26) is given by

$$
u_{0}(x, y)=1+\frac{1}{2} \frac{\mathrm{d} p_{0}}{\mathrm{~d} x}\left(y^{2}-h y\right)
$$

The pressure distribution in equation (27) can be found by utilizing the standard of preservation of mass and has the accompanying structure:

$$
\frac{\mathrm{d} p_{0}}{\mathrm{~d} x}=12 \frac{h(x)-\lambda_{0}}{h(x)^{3}} .
$$

By using equation (28) into equation (27), one can write the zero-order velocity distribution as

$$
u_{0}(x, y)=1+6\left(\frac{h-\lambda_{0}}{h^{3}}\right)\left(y^{2}-h y\right) \text {. }
$$

At the separation point located at $x=x_{s}$ and $y=\left(\bar{h}\left(x_{s}\right) / 2\right)$, as shown in Figure 2, the velocity goes to zero. Hence, from equation (29), we can write 


$$
x_{s}=\sqrt{3 \lambda_{0}-1}
$$

Integrating equation (28), write in the following form:

$$
p_{0}=12 \int_{x_{s}}^{x} \frac{h(x)-\lambda_{0}}{h(x)^{3}} \mathrm{~d} x
$$

where $x_{s}$ is the value of $x$ at $p=0$. Since we are assuming that the liquid splits evenly to the web and the roll, we can write $Q_{0}=2 U h_{c}$ and in dimensionless form $\lambda_{0}=\left(2 h_{c} / H_{0}\right)$. The pressure at the separation point may be found by the first integral of equation (28), using assumption $x_{b} \longrightarrow-\infty$, as

$$
p_{0}(x)=\frac{\left(6-(9 / 2) \lambda_{0}\right) x}{1+\left(x^{2} / 2\right)}-\frac{3 \lambda_{0} x}{\left(1+\left(x^{2} / 2\right)\right)^{2}}+\left(\frac{12}{\sqrt{2}}-\frac{9 \lambda_{0}}{\sqrt{2}}\right) \tan ^{-1} \frac{x}{\sqrt{2}}+\frac{6 \pi}{\sqrt{2}}\left(1-\frac{3 \lambda_{0}}{4}\right) .
$$

The simplest dynamic model of the separation region is based on the assumption that the film splits at the point, where $u_{0}=0$ and $p_{0}=0$; resultantly, the above equation becomes a transcendental equation in $\lambda_{0}$ whose solution may be obtained by a numerical technique named as the modified regula falsi method with a predefined accuracy of $10^{-10}$. For the said accuracy, the zero-order flow rate is $\lambda_{0}=1.3232524$, which is correct up to five decimal places, and the residual error in this case is 0.0000011085 ; this coating thickness has been achieved at the separation point $x_{s}=1.723298349$. It is worth to mention that the above zero-order results are more accurate as calculated by Middleman [17].

3.2. First-Order Problem and Its Solution. Equating the coefficients of terms We in equation (18), the first-order problem is

$$
\begin{gathered}
\frac{\mathrm{d} p_{1}}{\mathrm{~d} x}=\frac{\mathrm{d}^{2} u_{1}}{\mathrm{~d} y^{2}}+\frac{\mathrm{d}}{\mathrm{d} y}\left(\frac{\mathrm{d} u_{0}}{\mathrm{~d} y}\right)^{2}, \\
Q_{1}=\lambda_{1}=\int_{0}^{h(x)} u_{1} \mathrm{~d} y,
\end{gathered}
$$

with the agreeing first-order boundary conditions:

$$
\begin{aligned}
& u_{1}=0, \quad \text { at } y=0, \\
& u_{1}=0, \quad \text { at } y=h(x) .
\end{aligned}
$$

Adopting the same procedure as in Section 3.1, applying equation (27) into equation (33), after twice integration and using conditions (35), we acquire the following first-order solution:

$$
u_{1}(x, y)=\frac{1}{2} \frac{\mathrm{d} p_{1}}{\mathrm{~d} x}\left(y^{2}-h y\right)-\frac{1}{6}\left(\frac{\mathrm{d} p_{0}}{\mathrm{~d} x}\right)^{2}\left(2 y^{3}-3 h y^{2}+h^{2} y\right),
$$

$$
\frac{\mathrm{d} p_{1}}{\mathrm{~d} x}=-12 \frac{\lambda_{1}}{h^{3}}
$$

Making use of equations (28) and (37) into equation (36), the first-order velocity distribution takes the form

$$
\begin{aligned}
u_{1}(x, y) & =\frac{6 \lambda_{1}}{h^{3}}\left(h y-y^{2}\right)-24\left(\frac{h-\lambda_{0}}{h^{3}}\right)^{2}\left(2 y^{3}-3 h y^{2}+h^{2} y\right) \\
p(x) & =-\frac{9}{2} \frac{\lambda 1\left(\sqrt{2}\left(x^{2}+2\right)^{2} \arctan ((1 / 2) x \sqrt{2})+2 x^{3}+(20 / 3) x\right)}{\left(x^{2}+2\right)^{2}} .
\end{aligned}
$$

The film splits at the point where $p_{1}=0$; therefore, from the above pressure expression, one can get $\lambda_{1}=0$. From equation (39) and the separation point condition for the pressure, we can see clearly that the first-order flow rate is zero; therefore, in order to get more insight into Williamson's model, we need to take one more term of the perturbation analysis. 
3.3. Second-Order Problem and Its Solution. Equating the coefficients of terms We in equation (18), the second-order problem is

$$
\begin{aligned}
& \frac{\mathrm{d} p_{2}}{\mathrm{~d} x}=\frac{\mathrm{d}^{2} u_{2}}{\mathrm{~d} y^{2}}+2 \frac{\mathrm{d}}{\mathrm{d} y}\left(\frac{\mathrm{d} u_{0}}{\mathrm{~d} y} \frac{\mathrm{d} u_{1}}{\mathrm{~d} y}\right)^{2}, \\
& \mathrm{Q}_{2}=\lambda_{2}=\int_{0}^{h(x)} u_{1} \mathrm{~d} y,
\end{aligned}
$$

with the agreeing first-order boundary conditions:

$$
\begin{array}{ll}
u_{2}=0, & \text { at } y=0, \\
u_{2}=0, & \text { at } y=h(x) .
\end{array}
$$

Adopting a similar procedure, using equations (27) and (36) into equation (40) after twice integration and using the boundary conditions (42), we get the following second-order solution:

$$
\begin{aligned}
u_{2}(x, y)= & \frac{24}{h^{3}}\left(\frac{\mathrm{d} p_{0}}{\mathrm{~d} x}\right)^{3}\left(3 y^{4}-6 h y^{3}+4 h^{2} y^{2}\right)+\frac{1}{2} \frac{\mathrm{d} p_{2}}{\mathrm{~d} x}\left(y^{2}-h y\right) \\
& -\frac{1}{2}\left(\frac{576\left(h^{3}-\lambda_{0}^{3}\right)-1728\left(h^{2} \lambda_{0}-h \lambda_{0}^{2}\right)}{h^{6}}\right), \\
\frac{\mathrm{d} p_{2}}{\mathrm{~d} x}= & -\frac{12}{5}\left(\frac{5 h^{4} \lambda_{2}+96\left(h^{3}-\lambda_{0}^{3}\right)-288\left(h^{2} \lambda_{0}-h \lambda_{0}^{2}\right)}{h^{7}}\right) .
\end{aligned}
$$

By substituting the first- and second-order pressure gradient distributions into equation (43), one can find the third-order velocity distribution. The third-order pressure distribution at the separation point may be found by integrating the above equation as $x_{b} \longrightarrow-\infty$ given by

$$
p_{2}(x)=\left[\begin{array}{c}
2.25 \sqrt{2}\left(\lambda_{2}-0.142\right)\left\{-2 \tan ^{-1}\left(\frac{x}{\sqrt{2}}\right)-\pi\right\}- \\
\left\{\begin{array}{c}
\left(\lambda_{2}-0.142\right) x^{11}+\left((34 / 3) \lambda_{2}-0.142\right) x^{9}+\left((152 / 3) \lambda_{2}-7.5\right) x^{7}+\left(112 \lambda_{2}-76.74\right) x^{5} \\
+\left((368 / 3) \lambda_{2}-1.67\right) x^{3}+\left((160 / 3) \lambda_{2}-49.15\right) x
\end{array}\right.
\end{array}\right] .
$$

From equation (43), a relation between the separation point and third-order flow rate can be found. Setting $x=x_{s}$ and $y=\left(\bar{h}\left(x_{s}\right) / 2\right)$, in order to find the third-order separation point, the regula falsi method has been used with a predefined tolerance of $10^{-10}$ which results in $\lambda_{2}=0.315$, and the resulting third-order separation point is $x_{s}=0.2475$. Maple has a Numerical Analysis package that implements many of the techniques we will discuss. The results till the third-order approximation are tabulated in Table 1.

Combining the solutions at each order of approximation yield the solutions up to second order for velocity, pressure gradient, and pressure.

\section{Operating Variables}

Once the velocity, pressure distribution, and pressure gradient are found, then all other interesting engineering quantities are readily available. The operating variables are computed in the following manner.
4.1. Coating Thickness. The substrate thickness $H_{b}$ at the entering is given by

$$
H_{b}=1+\frac{x_{b}^{2}}{2}
$$

It is a very large number, in the case of an infinite reservoir.

Since we are assuming that fluid separates uniformly to coat both the roll and the plane, we can write volumetric flow rate in the dimensional form as $Q=2 U H$ and

$$
\frac{H}{H_{0}}=\frac{\lambda}{2} \text {. }
$$

This gives the resulting leaving coating thickness in a dimensionless form.

The examination is yet incomplete at this point as the relationship between the nondimensional flow rate $\lambda$ and the separation point $x_{s}$ needs to be evaluated. Figure 2 
TABLE 1: Effect of Weissenberg number on operating variables.

\begin{tabular}{lccccc}
\hline We & $\lambda$ & $H / H_{0}$ & $x_{s}$ & $F$ & $P_{w}$ \\
\hline 0.01 & 1.3232 & 0.6616 & 2.4171 & 0.02258 & -5.06243 \\
0.1 & 1.3264 & 0.6632 & 2.4434 & 0.00302 & -4.70615 \\
0.2 & 1.3358 & 0.6679 & 2.4874 & -0.03125 & -4.12705 \\
0.3 & 1.3516 & 0.6758 & 2.5486 & -0.08286 & -3.15219 \\
0.4 & 1.3736 & 0.6868 & 2.6263 & -0.15480 & -1.54170 \\
0.5 & 1.4020 & 0.7010 & 2.7191 & -0.25030 & 1.02603 \\
0.6 & 1.4366 & 0.7183 & 2.8248 & -0.37213 & 4.96889 \\
0.7 & 1.4776 & 0.7388 & 2.9409 & -0.52245 & 10.8158 \\
0.8 & 1.5248 & 0.7624 & 3.0646 & -0.70248 & 19.2218 \\
0.9 & 1.5784 & 0.7892 & 3.1804 & -0.89035 & 29.6293 \\
0.99 & 1.6382 & 0.8191 & 3.2653 & -0.89219 & 32.4523 \\
\hline
\end{tabular}

indicates that the web separates equally, and, consequently, the segregation point is $\left(x_{s}, h\left(x_{s}\right) / 2\right)$; here, the pressure and velocity profile approach zero. This is assumed as the roll and sheet has equal velocity. It causes segregation point $x_{s}$ depending upon the dimensionless coating thickness $\lambda$.

In case of Newtonian fluid, the relationship between segregation point $x_{s}$, nondimensional flow rate, and the rheological parameters admits the value of one of these parameters depending on others. As in case of Williamson fluid, it is difficult to calculate explicitly $x_{s}$ in terms of $\lambda$. To find the segregation point $x_{s}$, some numerical system is needed.

4.2. Roll Separating Force. The roll separating force $F$ is given by

$$
F=\int_{-\infty}^{x_{s}} p(x) \mathrm{d} x
$$

where $F=\left(\bar{F} H_{0} / \eta_{0} U R W\right), \bar{F}$ is the dimensional roll separating force per unit width $W$.

4.3. Power Contribution. The power transmitted to the fluid by the roll is calculated by integrating the product of shear stress and the roll surface speed over the roll surface which is obtained by setting $\bar{y}=H_{0}$, as

$$
P_{w}=\int_{-\infty}^{x_{s}} \tau_{x y}(x, 1) \mathrm{d} x .
$$

Here, $P_{w}=\left(\overline{P_{w}} / \eta_{0} W U^{2}\right)$ is the nondimensional power and $\tau_{x y}=\left(\bar{\tau}_{x y} H_{0} / \eta_{0} U\right)$ the nondimensional stress tensor given by equation (14).

4.4. Adiabatic Temperature. The power contribution has the capability to increase the temperature of fluid to the maximum, given by rise in adiabatic temperature $(\Delta T)_{\text {ave }}$ :

$$
(\Delta T)_{\text {ave }}=\frac{P_{w}}{Q \rho C_{p}},
$$

where $C_{p}$ is the melt heat capacity at constant pressure.

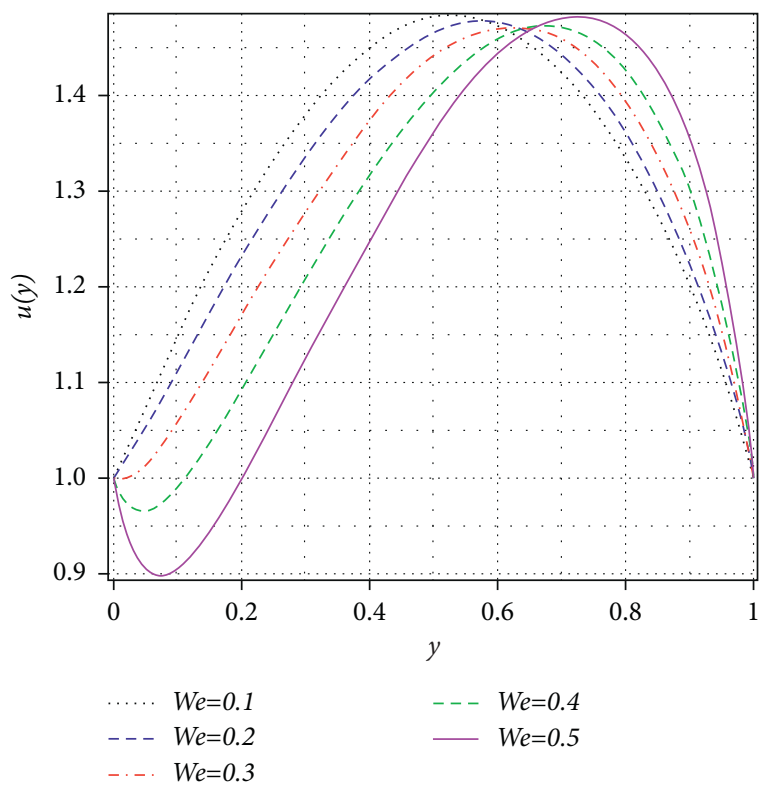

FIGURE 3: Effect of the Weissenberg number on velocity distribution at nip region $(x=0)$.

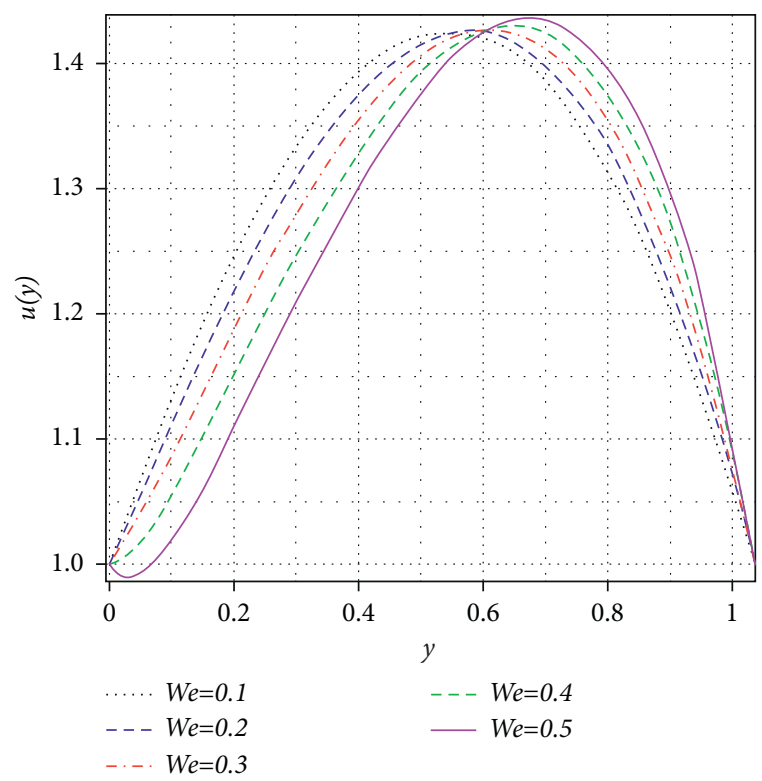

FIGURE 4: Effect of the Weissenberg number on velocity distribution at $x=0.25$.

\section{Results and Discussions}

This article investigates the roll coating procedure for an incompressible Williamson fluid. For the simplification of motion equations, the LAT is applied. Maple 15 software has been used to generate numerical results for the volumetric flow rate $\lambda$, the separation point $x_{s}$, the exit sheet thickness $H / H_{0}$, the power contribution, and the roll separating force presented in Table 1 for various values of the Weissenberg number We. The maximum coating thickness has been observed as 0.8200 by approaching $\mathrm{We} \longrightarrow 1$, and for this 

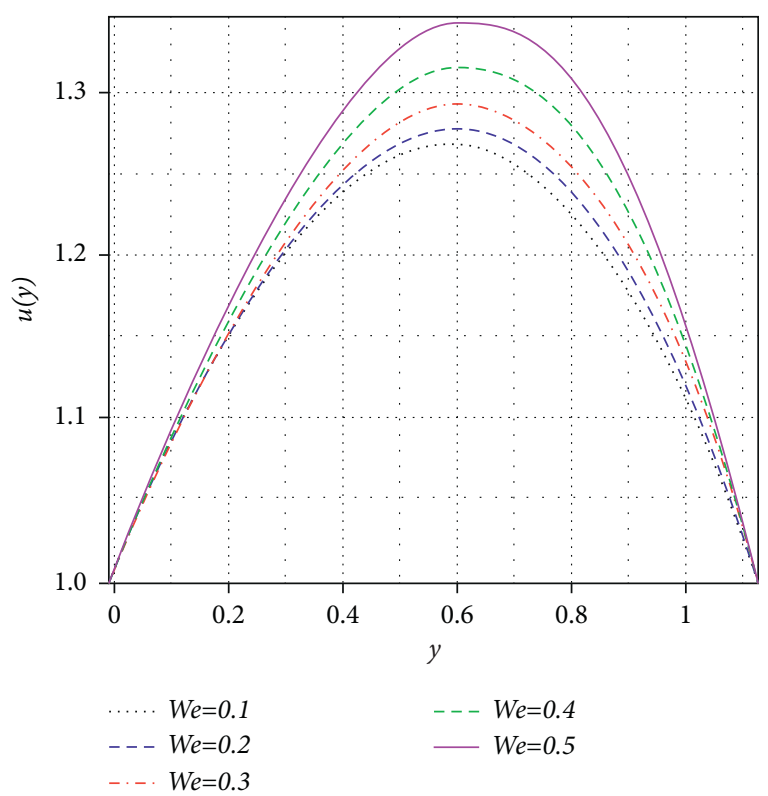

FIGURE 5: Effect of the Weissenberg number on velocity distribution at $x=0.5$.

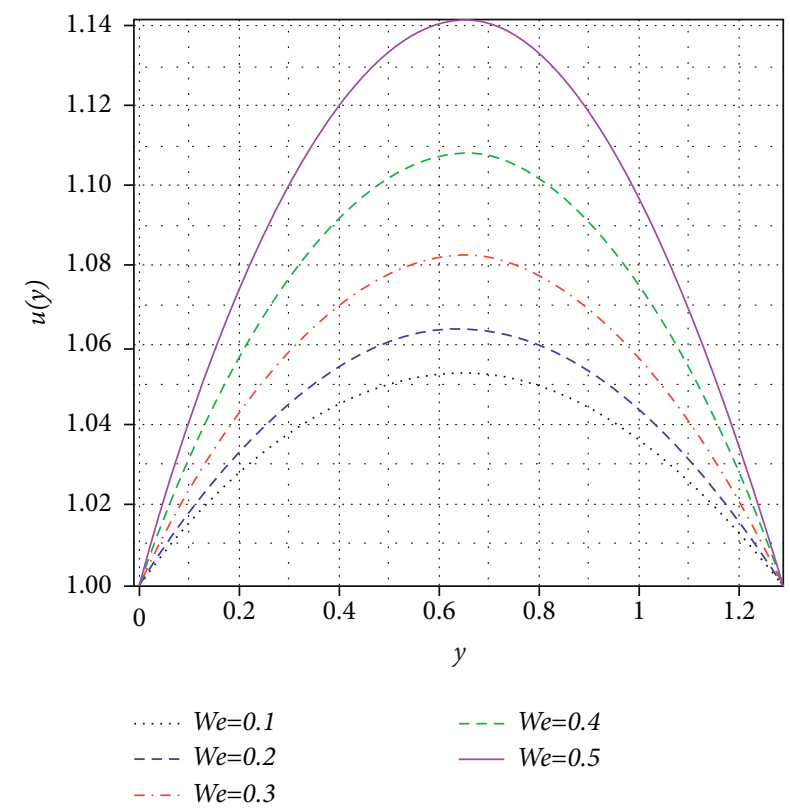

FIGURE 6: Effect of the Weissenberg number on velocity distribution at $x=0.75$.

coating thickness, the optimum point of separation is detected. It is noticed that an increase in We causes an increase in the coating thickness. The minimum coating thickness when $\mathrm{We} \longrightarrow 0$ has been observed as 0.6610 . The coating thickness, volume flow rate, and separation point are increasing functions of We (forces of inertia are controlling viscous force).

The outcome for the dimensionless velocity profiles is projected in Figures 3-10. Through the variation of We, velocity profiles at various locations of the roll coating

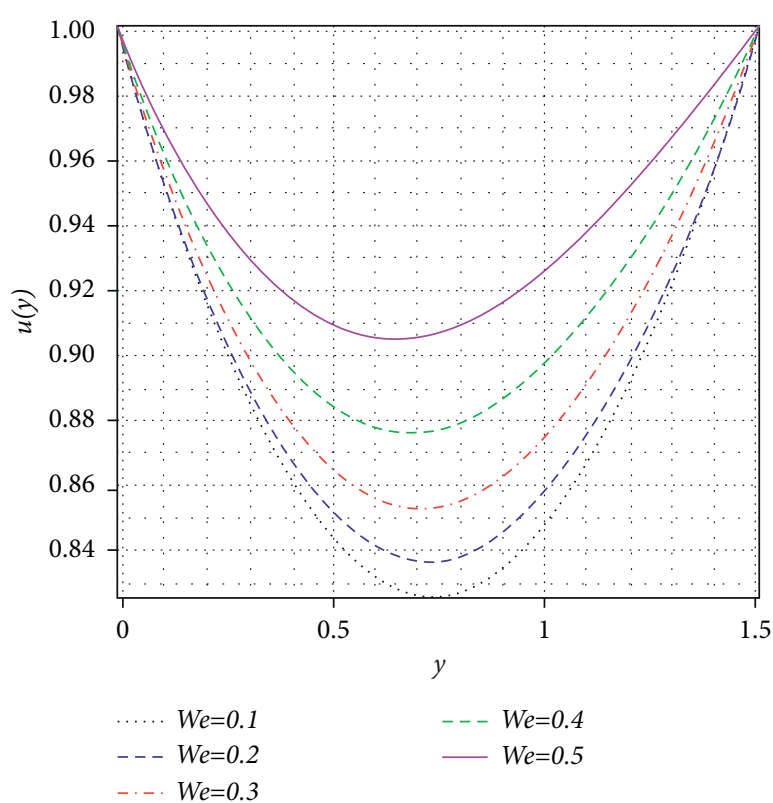

FIgURE 7: Effect of the Weissenberg number on velocity distribution at $x=1$.

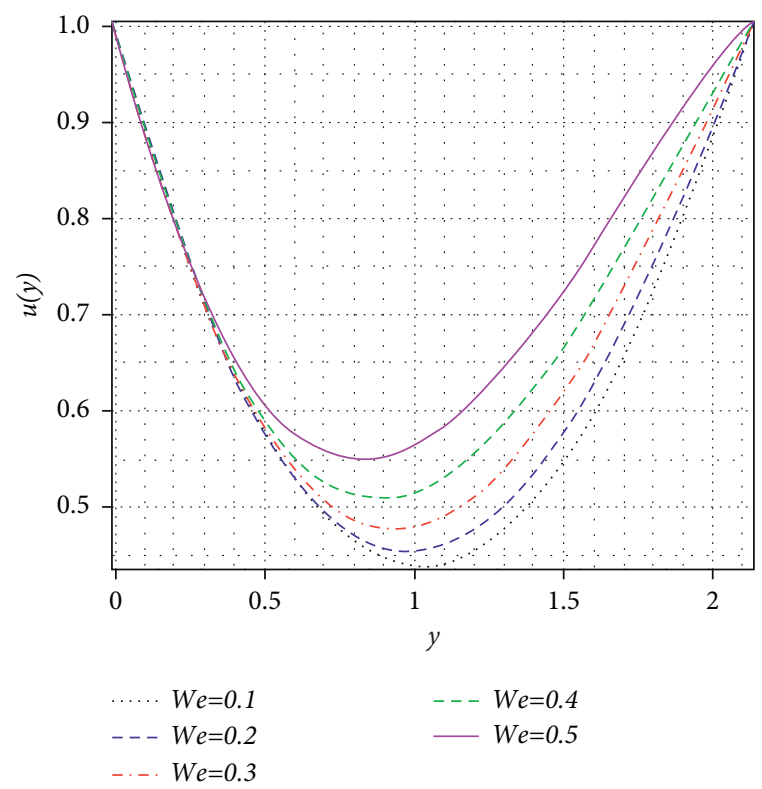

FIgURE 8: Effect of the Weissenberg number on velocity distribution at $x=1.5$.

procedure are presented. Figures 3 and 4 are plotted at positions $x=0$ and $x=0.25$, respectively, indicating that the increase in We first causes the velocity of the fluid to decrease in $0<y<0.602$ and then increase monotonically beyond 0.6 .

It is interesting to see from Figure 3 that, at the nip region, the reverse flow has been observed for the higher values of $\mathrm{We}$, and as the material moves away from the nip in the direction of separation point, the backward flow decreases as shown graphically in Figures 4-6 and the fluid 


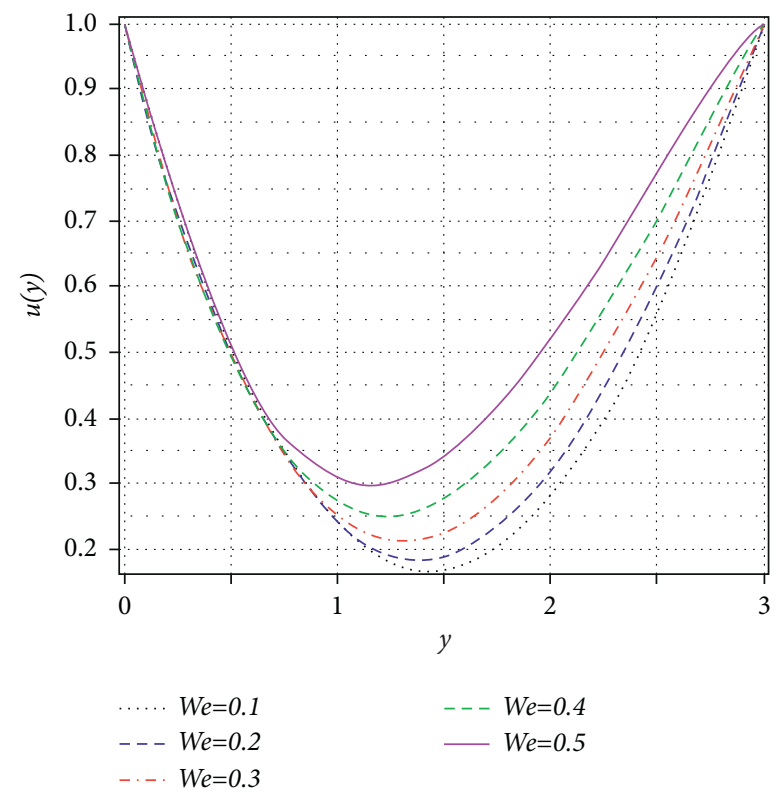

FIGURE 9: Effect of the Weissenberg number on velocity distribution at $x=2$.

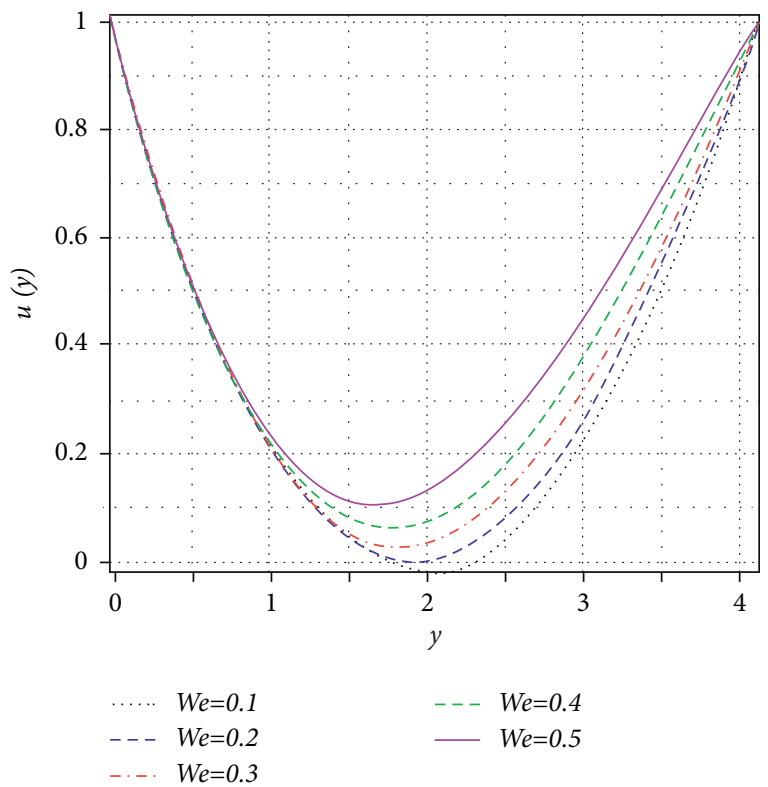

FIGURE 10: Effect of the Weissenberg number on velocity distribution at $x=2.5$.

becomes more developed. Figure 4 indicates that all the velocity curves have the same point of intersection, that is, $(y=0.6, u=1.4263)$ for different values of the Weissenberg number.

Figures 5 and 6 have been depicted at positions $x=0.5$ and 0.75 , respectively; here, one can observe that an increase in We causes increase in the velocity profile as We is the ratio of viscous forces to the elastic forces. It indicates that the viscous forces become controlling forces once the web reaches the position $x>0.25$. It is also observed from these graphs that fluid becomes fully developed when one moves toward the separation point.

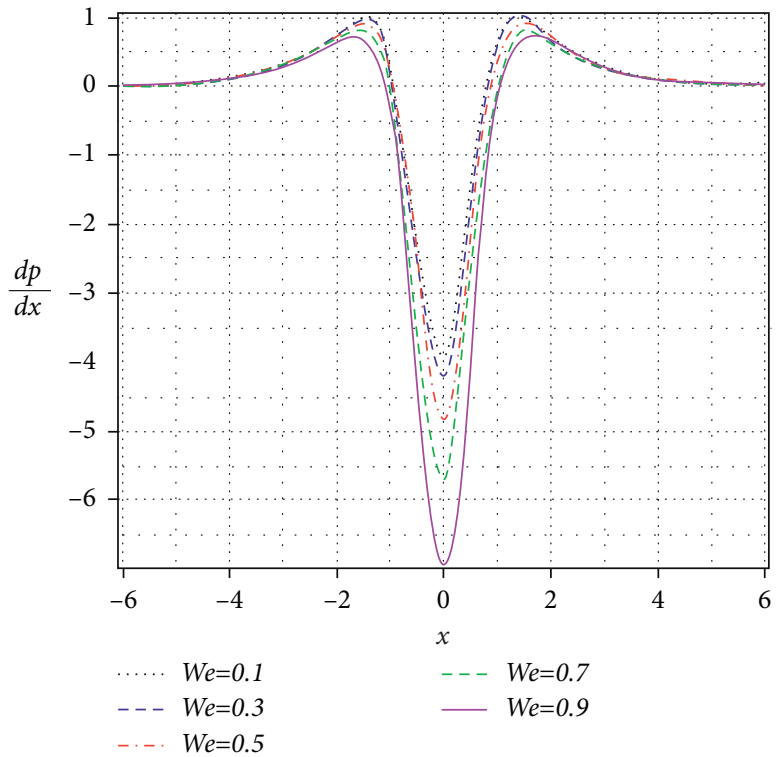

FIGURE 11: Effect of the Weissenberg number on pressure gradient distribution.

Comparative to Figures 5 and 6, a symmetric but opposite behavior has been observed in Figures 7-10, which are sketched at $x=1, x=1.5, x=2$, and $x=2.5$, respectively. With an increase in the Weissenberg number, the velocity distribution increases. It is also highlighted that, as the fluid approaches the separation point, the fluid viscosity increases and dominance of viscous force over elastic force will cause coating on the web; this behavior can clearly been seen from Figures 7-10, Furthermore, from these figures, one can analyze that the gap between the velocity lines near to the web is smaller as compared to the gab between the velocity line near to the roll. 


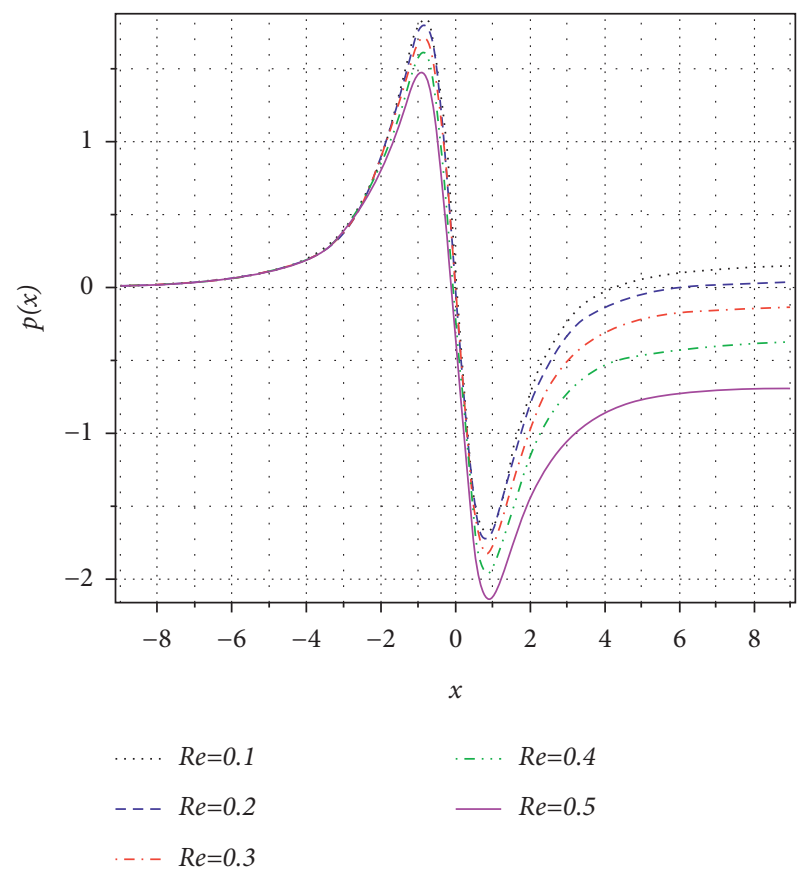

Figure 12: Effect of the Weissenberg number on pressure distribution.

The result for the dimensionless pressure gradient distributions is presented in Figure 11, whereas the outcomes for the dimensionless pressure profile are projected in Figure 12. It is observed that the pressure gradient rises by increasing the Weissenberg number, whereas the pressure distribution decreases by increasing the Weissenberg number. Starting from zero pressure at the attachment point, pressure starts increasing and attains its extreme values right at $x= \pm 0.86$.

\section{Conclusion}

Using lubrication approximation theory, the system of nonlinear partial differential equations is reduced to ordinary differential equations. Then, the obtained nonlinear ODEs are linearized using the regular perturbation technique. The influence of various evolving physical parameters on velocity components, flow rate, pressure profile, roll separating force, power contribution, separation point, and most important coating thickness have been studied theoretically. Some of the results are tabulated numerically and some are presented graphically.

The key findings of the present study are as follows:

(i) For the pseudoplastic fluids, the results by Middleman [17] were verified when $\mathrm{We} \longrightarrow 0$ and extended

(ii) Weissenberg's number acts as a controlling parameter for the rate of flow, thickness in coating, power contribution, pressure, roll separating force, and separation point

(iii) The extreme values for the pressure distribution have been found at $x= \pm 0.86$ (iv) Viscous force plays a vital and dominating role in coating thickness, force of separation, power, and pressure profile

(v) The reverse flow has been observed at the nip region for the higher values of $\mathrm{We}$, and as the material moves away from the nip in the direction of separation point, the backward flow decreases

(vi) It is worth mentioning that the above zero-order results are more accurate as calculated by Middleman

\section{Data Availability}

The data and materials used in this work are included in the manuscript.

\section{Conflicts of Interest}

The authors declare that they have no conflicts of interest.

\section{Authors' Contributions}

All the authors contributed equally.

\section{Acknowledgments}

S. Saleem and A. Al-Zubaidi extend their appreciation to the Deanship of Scientific Research at King Khalid University for funding this work through the research group program under Grant no. RGP. 1/324/42.

\section{References}

[1] O. A. Abegunrin, S. O. Okhuevbie, and I. L. Animasaun, "Comparison between the flows of two non-Newtonian fluids over an upper horizontal surface of paraboloid of revolution: boundary layer analysis," Alexandria Engineering Journal, vol. 55, no. 3, pp. 1915-1929, 2016.

[2] O. A. Abegunrin and I. L. Animasaun, "Motion of Williamson fluid over an upper horizontal surface of a paraboloid of revolution due to partial slip and buoyancy: boundary layer analysis," Defect and Diffusion Forum, vol. 378, pp. 16-27, 2017.

[3] N. A. Shah, I. L. Animasaun, J. D. Chung et al., "Significance of nanoparticle's radius, heat flux due to concentration gradient, and mass flux due to temperature gradient: the case of water conveying copper nanoparticles," Scientific Reports, vol. 11, no. 1, p. 1882, 2021.

[4] I. L. Animasaun and A. J. Omowaye, "Upper-convected maxwell fluid flow with variable thermo-physical properties over a melting surface situated in hot environment subject to thermal stratification," Journal of Applied Fluid Mechanics, vol. 9, no. 4, pp. 1777-1790, 2016.

[5] K. S. Mekheimer, B. M. Shankar, S. F. Ramadan, H. E. Mallik, and M. S. Mohamed, "On the stability of convection in a nonnewtonian vertical fluid layer in the presence of gold nanoparticles: drug agent for thermotherapy," Mathematics, vol. 9, no. 11, p. 1320, 2021.

[6] A. Z. Zaher, A. M. A. Moawad, K. S. Mekheimer, and M. M. Bhatti, "Residual time of sinusoidal metachronal ciliary flow of non-newtonian fluid through ciliated walls: 
fertilization and implantation," Biomechanics and Modeling in Mechanobiology, vol. 20, no. 2, pp. 609-630, 2021.

[7] S. R. El Koumy, E. S. I. Barakat, and S. I. Abdelsalam, "Hall and porous boundaries effects on peristaltic transport through porous medium of a maxwell model," Transport in Porous Media, vol. 94, no. 3, pp. 643-658, 2012.

[8] S. I. Abdelsalam, K. S. Mekheimer, and A. Z. Zaher, "Alterations in blood stream by electroosmotic forces of hybrid nanofluid through diseased artery: aneurysmal/stenosed segment," Chinese Journal of Physics, vol. 67, pp. 314-329, 2020.

[9] R. Raza, F. Mabood, R. Naz, and S. I. Abdelsalam, “Thermal transport of radiative Williamson fluid over stretchable curved surface," Thermal Science and Engineering Progress, vol. 23, no. 1, Article ID 100887, 2021.

[10] D. V. Lyubimov and A. V. Perminov, "Motion of a thin oblique layer of a pseudoplastic fluid," Journal of Engineering Physics and Thermophysics, vol. 75, no. 4, pp. 920-924, 2002.

[11] R. V. Williamson, "The flow of pseudoplastic materials," Industrial \& Engineering Chemistry, vol. 21, no. 11, pp. 1108-1111, 1929.

[12] F. Ali, Y. Hou, M. Zahid, and M. Rana, "Mathematical analysis of pseudoplastic polymers during reverse roll-coating," Polymers, vol. 12, no. 10, p. 2285, 2020.

[13] S. Nadeem and N. S. Akbar, "Numerical solutions of peristaltic flow of Williamson fluid with radially varying MHD in an endoscope," International Journal for Numerical Methods in Fluids, vol. 66, no. 2, pp. 212-220, 2011.

[14] S. D. Cramer and J. M. Marchello, "Numerical evaluation of models describing non-newtonian behavior," AIChE Journal, vol. 14, no. 6, pp. 980-983, 1968.

[15] J. H. Taylor and A. C. Zeltlemoyer, "Hypothesis on the mechanism of ink splitting during printing," TAPPI Journal, vol. 41 , no. 12 , pp. $749-757,1958$.

[16] J. C. Hintermaier and R. E. White, "The splitting of water film between rotating rolls," TAPPI Journal, vol. 48, no. 11, pp. 617-625, 1965.

[17] Y. Greener and S. Middleman, "A theory of roll coating of viscous and viscoelastic fluids," Polymer Engineering and Science, vol. 15, no. 1, pp. 1-10, 1975.

[18] H. Benkreira, M. F. Edwards, and W. L. Wilkinson, "A semiempirical model of the forward roll coating flow of newtonian fluids," Chemical engineering Science, vol. 36, no. 2, pp. 423-427, 1981.

[19] S. Sofou and E. Mitsoulis, "Roll-over-web coating of pseudoplastic and viscoplastic sheets using the lubrication approximation," Journal of Plastic Film \& Sheeting, vol. 21, no. 4, pp. 307-333, 2005.

[20] M. Zahid, T. Haroon, M. Rana, and A. Siddiqui, "Roll coating analysis of a third grade fluid," Journal of Plastic Film \& Sheeting, vol. 33, no. 1, pp. 72-91, 2017.

[21] M. Zafar, M. A. Rana, M. Zahid, and B. Ahmad, "Mathematical analysis of the coating process over a porous web lubricated with upper-convected maxwell fluid," Coatings, vol. 9, no. 7, p. 458, 2019.

[22] M. Renardy, "High Weissenberg number boundary layers for the upper convected maxwell fluid," Journal of Non-Newtonian Fluid Mechanics, vol. 68, no. 1, pp. 125-132, 1997.

[23] M. Mohamadali and N. Ashrafi, "Similarity solution for high Weissenberg number flow of upper-convected maxwell fluid on a linearly stretching sheet," Journal of Engineering, vol. 2016, Article ID 9718786, 10 pages, 2016.

[24] I. Daprà and G. Scarpi, "Perturbation solution for pulsatile flow of a non-Newtonian Williamson fluid in a rock fracture," International Journal of Rock Mechanics and Mining Sciences, vol. 44, no. 2, pp. 271-278, 2007. 